'Servicio de Medicina, Sección de Nefrología, Hospital Naval Almirante Nef. Viña del Mar, Chile.

2Departamento de Medicina, Escuela de Medicina, Universidad de Valparaíso. Viña del Mar, Chile.

${ }^{3}$ Servicio de Pediatría, Hospital Dr. Gustavo Fricke. Viña del Mar, Chile.

${ }^{4}$ Departamento de Anatomía Patológica, Facultad de Medicina, Pontificia Universidad Católica de Chile. Santiago, Chile.

Trabajo no recibió financiamiento. Los autores declaran no tener conflictos de interés.

Recibido el 31 de octubre de 2019, aceptado el 12 de mayo de 2020.

Correspondencia a: Dr. Jorge Vega 5 Norte 1035, Viña del Mar, Chile. jvegastieb@gmail.com

\section{Nefropatía por C1q: una rara variedad de síndrome nefrótico. Caso clínico}

\author{
JORGE VEGA ${ }^{1,2}$, ELISA GUTIÉRREZ ${ }^{3}$, \\ GONZALO P. MÉNDEZ ${ }^{4}$, LUIS BOFILL ${ }^{3}$
}

\section{C1q nephropathy. Report of one case}

C1q nephropathy is a rare glomerulopathy characterized by mesangial deposition of the complement component C1q. These deposits can be isolated or associated with immunoglobulins or complement fractions, which are observed by immunofluorescence or immunohistochemical microscopy. In ultramicroscopy, dense mesangial deposits and alterations of the podocyte are observed. Clinically it presents as a nephrotic syndrome (NS) or by alterations of the urinalysis such as proteinuria and/or hematuria in children and young adults. In light microscopy, it is expressed with a morphological pattern of minimal change disease (MCD), mesangial proliferative glomerulonephritis or focal segmental glomerulosclerosis (FSGS). The NS during its evolution usually evolve in steroid resistance or steroid dependency, often requiring the association of immunosuppressants to obtain remission. We report a 14 years old male with a history of NS and its evolution under various treatments during a 12-year follow-up.

(Rev Med Chile 2020; 148: 702-706)

Key words: Complement System Proteins; Glomerulonephritis; Nephrosis, Lipoid; Nephrotic Syndrome; Proteinuria.
$\mathrm{E}$ n pacientes con síndrome nefrótico $(\mathrm{SN})$ primario, cuando se efectúa una biopsia renal (BpR) lo más frecuente es encontrar una nefropatía membranosa en adultos o de cambios mínimos (NCM) en niños. Con menos frecuencia una glomeruloesclerosis focal y segmentaria (GSFS), o una glomerulonefritis (GN) membranoproliferativa (MP).

En 1985 Jennette y cols describieron una glomerulopatía caracterizada por depósitos mesangiales de $\mathrm{Clq}$ en la inmunofluorescencia, en forma dominante o codominante, con inmunoglobulinas y/o fracciones del complemento y la existencia de depósitos densos mesangiales en la microscopía electrónica. Los criterios de exclusión fueron la evidencia clínica o serológica de un lupus eritematoso (LES) o GNMP tipo 1. A esta nueva entidad propusieron llamarla "nefropatía por C1q" (NC1q). Usualmente se manifestaba como $\mathrm{SN}$ resistente a corticoides ${ }^{1}$. Desde entonces se han publicado series y casos clínicos, que han mostrado que esta entidad tiene un comportamiento distinto al SN primario clásico ${ }^{2-19}$. Otros autores, en cambio, creen que el depósito de C1q es un marcador inespecífico del tráfico de proteínas y que esta entidad está dentro del espectro clínico de las podocitopatías difusas (NCM/GSFS) ${ }^{19}$.

La prevalencia de la NC1q es muy baja, variando entre 0,2 a 2,5\% en BpR de niños y adultos; 2,1 a $6 \%$ en biopsias pediátricas y hasta $16,5 \%$ en biopsias de niños con $\mathrm{SN}^{3}$. En Revista Médica de Chile solo hay una publicación en un paciente extranjero ${ }^{10}$.

\section{Caso clínico}

Varón de 14 años que a la edad de 2 años y 3 meses presentó un SN. Había edema generalizado, ascitis, oliguria, hipoalbuminemia (1,6 g/dl), hiperlipidemia (colesterol: $494 \mathrm{mg} / \mathrm{dl}$, triglicéridos: 
$656 \mathrm{mg} / \mathrm{dl}$ ), hematuria microscópica (2-4 eritrocitos por campo) y proteinuria de $8 \mathrm{~g} / 24 \mathrm{~h}$ (217 $\mathrm{mg} / \mathrm{m}^{2} / \mathrm{h}$ ). La presión arterial fue normal (97/56 $\mathrm{mmHg}$ ) y no existía disfunción renal (uremia:14 $\mathrm{mg} / \mathrm{dl}$, creatinina: $0,3 \mathrm{mg} / \mathrm{dl}$ ). La hemoglobina fue: $14,1 \mathrm{~g} / \mathrm{dl}$, leucocitos: $10.500 \mathrm{~mm}^{3}$, plaquetas: $394.000 \mathrm{~mm}^{3}$ y VHS: $125 \mathrm{~mm} / \mathrm{h}$.

Se prescribió prednisona (PDN) $2 \mathrm{mg} / \mathrm{kg} /$ día, desapareciendo la proteinuria al sexto día, reduciendo los esteroides progresivamente hasta suspenderlos en 4 meses. Meses más tarde presentó una recaída, remitiendo la proteinuria a las 2 semanas de reiniciar la PDN.

En los 5 años siguientes sufrió numerosas recaídas, a menudo precedidas de infecciones respiratorias, que remitían con PDN pero con dependencia de los esteroides en días alternos. Para reducir efectos secundarios, cuando tenía 7 años de edad, se indicó ciclofosfamida $2 \mathrm{mg} / \mathrm{kg}$ durante 8 semanas asociado a PDN $5 \mathrm{mg} \mathrm{c} / 48 \mathrm{~h}$, con lo cual se obtuvo remisión completa, la que duró 18 meses.

Presentó una nueva recaída a los 8 años, que remitió con PDN $2 \mathrm{mg} / \mathrm{kg}$ y evolucionó con dependencia a altas dosis de PDN en días alternos. Por ello, a los $91 / 2$ años, se inició micofenolato mofetil (MMF) $1.250 \mathrm{mg} /$ día $\left(600 \mathrm{mg} / \mathrm{m}^{2} /\right.$ día $)$ (pesaba $34 \mathrm{~kg}$ ), permitiendo reducir la dosis de PDN y mantener la remisión. A los 11 años, presentó otra recaída, agregándose ciclosporina (CsA) $(5,2 \mathrm{mg} / \mathrm{kg} /$ día, en dosis inicial) al esquema previo, obteniendo la remisión completa. Los niveles de complemento $\mathrm{C} 3$ y $\mathrm{C} 4$ fueron normales y los ANA $y$ anticuerpos anti-DNA negativos. En los 3 años siguientes, estando en tratamiento con MMF, CsA y PDN ( $10 \mathrm{mg} \mathrm{c} / 48 \mathrm{~h}$ ) presentó 10 recaídas de su $\mathrm{SN}$, respondiendo rápidamente al incremento en las dosis de PDN.

Al cumplir 14 años aceptó que se efectuara una BpR (previamente sus padres se habían negado). La evaluación de laboratorio previa mostró: hemoglobina: $13 \mathrm{~g} / \mathrm{dl}$, leucocitos: $8.200 \mathrm{~mm}^{3}$, plaquetas: $234.000 \mathrm{~mm}^{3}$, VHS: $44 \mathrm{~mm} / \mathrm{h}$, creatinina: 0,37 $\mathrm{mg} / \mathrm{dl}$, albuminemia: 4,0 g/dl, colesterol: $226 \mathrm{mg} /$ dl, triglicéridos: $181 \mathrm{mg} / \mathrm{dl}$, C3: $90 \mathrm{mg} / \mathrm{dl}$ (70-140), C4: $26 \mathrm{mg} / \mathrm{dl}$ (10-40), anticuerpos antinucleares y anti-DNA negativos, IgG: $371 \mathrm{mg} / \mathrm{dl}$ (680-1548), IgA: 142 mg/dl (81-252), IgM: 82 mg/dl (45-256).

La BpR mostró un patrón morfológico en la inmunofluorescencia y microscopía electrónica compatible con una NC1q (Tabla 1, Figuras 1 y 2).

\section{Tabla 1. Biopsia renal en nefropatía C1q}

Microscopía de luz
18 glomérulos, ninguno obsoleto
1 glomérulo con cambios isquémicos leves
Túbulos con atrofia focal leve (5-10\%)
No hay fibrosis intersticial ni infiltrados
Vasos de estructura conservada
Inmunofluorescencia
15 glomérulos, ninguno esclerosado
Depósitos mesangiales de carácter segmentario de C1q (2
a $3+/ 4+$ ), IgG ( $2+/ 4+$ ), IgM (trazas)
No hay reactividad para IgA ni C'3 en glomérulos, vasos,
túbulos ni intersticio
Microscopía electrónica
Depósitos densos mesangiales finos bajo la membrana
basal
Asas capilares periféricas y membrana basal conservan su
estructura
Existe un defecto electrón lúcido intramembranoso
No hay depósitos subendoteliales ni subepiteliales
Los podocitos presentan borramiento pedicelar completo,
condensación de filamentos sobre la membrana basal y
transformación microvellosa
No hay inclusiones tubuloreticulares en endotelio glo-
merular

A pesar de lo tórpido de la evolución de su glomerulopatía, solo se hospitalizó 3 veces, pudiendo acudir al colegio normalmente, teniendo un excelente rendimiento académico. Si bien a los 15 años tiene talla baja, no desarrolló otros efectos secundarios al uso prolongado a corticoides como hipertensión, cataratas u osteoporosis ni tampoco desarrolló una depresión. Los padres del paciente autorizaron la comunicación del caso clínico.

\section{Discusión}

Para el diagnóstico de NC1q se requiere la presencia de $\mathrm{Clq}$ en el mesangio con una intensidad $\geq 2+$ (en una escala de $0+$ a $4+$ ) en la microscopía de inmunofluorescencia o inmunohistoquímica, la que puede ser aislada o acompañarse de inmunoglobulinas u otras fracciones del complemento en forma codominante, además de la presencia de depósitos densos mesangiales y/o paramesangiales 
Figura 1. Zona cortical renal de arquitectura conservada, sin signos de atrofia o fibrosis. Los dos glomérulos presentes conservan su arquitectura, sin evidencias de lesiones esclerosantes ni proliferativas. (Tinción de PAS; 200x).
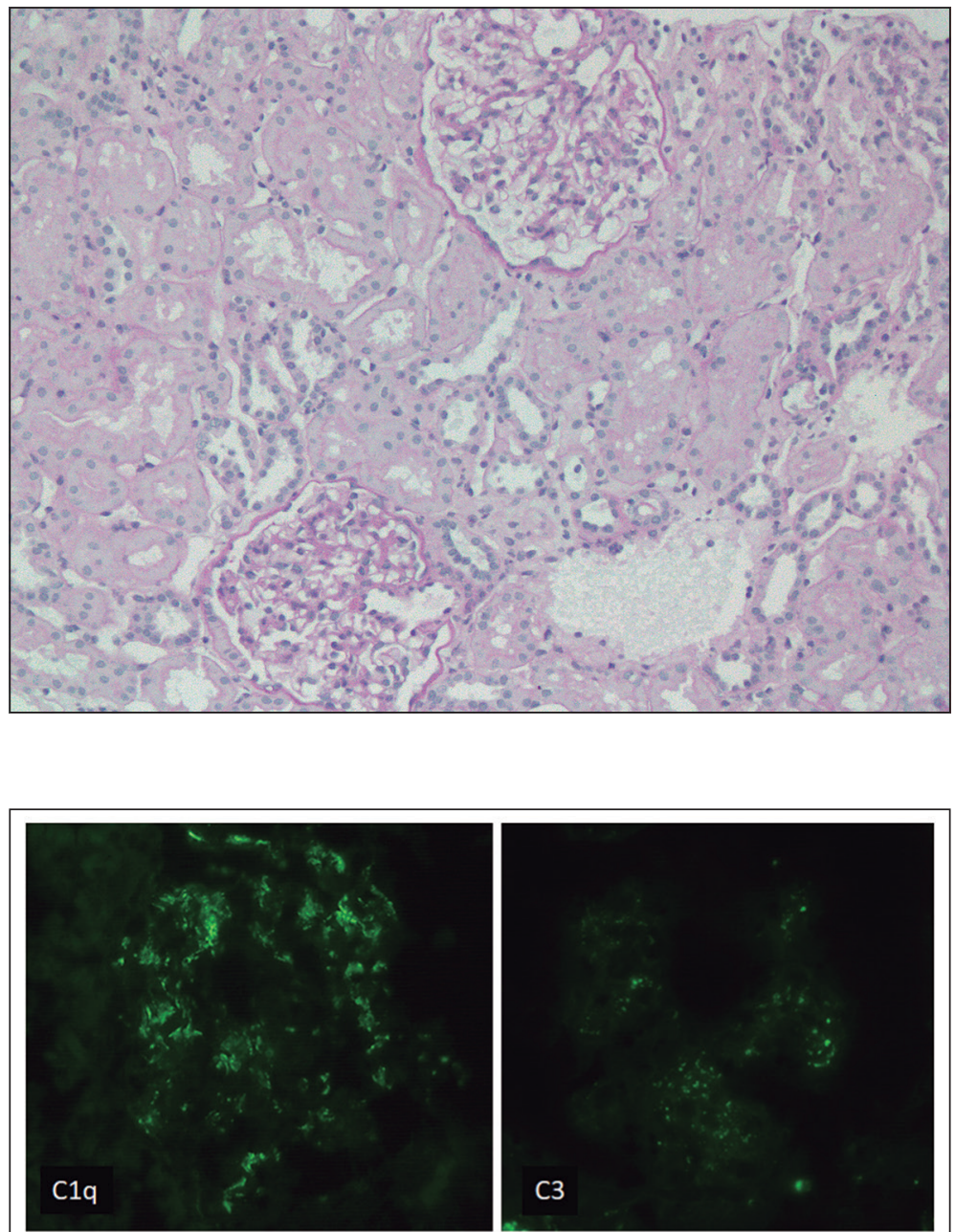

Figura 2. Microscopía de inmunofluorescencia. Se observa manifiesto predominio de los depósitos mesangiales de C1q por sobre C3, IgG e IgM.
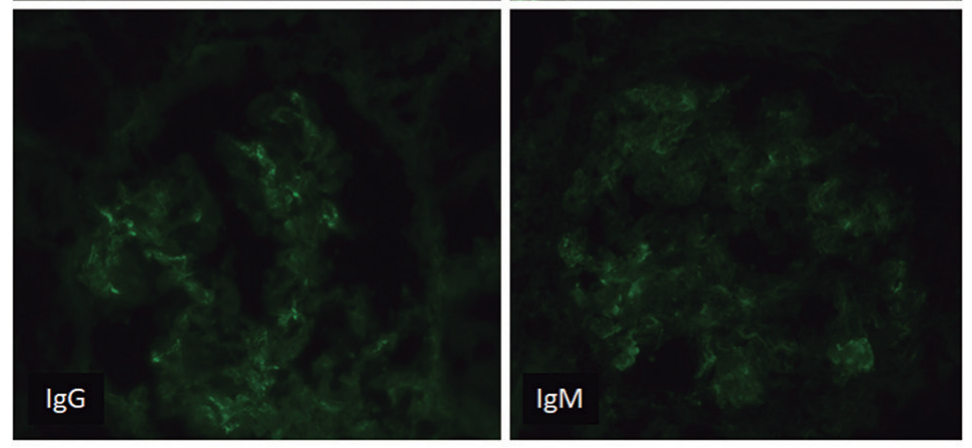

en conjunto con alteraciones pedicelares significativas $^{2,3,11,12,16}$. En este paciente la microscopía óptica mostró un patrón tipo NCM, la inmunofluorescencia depósitos de C1q de intensidad 2+ a 3+ con menor co-expresión de IgG y la ultraestructura, depósitos densos mesangiales y alteraciones podocitarias propias del SN, todo ello compatible con esta variedad de glomerulopatía.

La presentación clínica más frecuente es un SN, como en este paciente, o alteraciones asintomá- 
ticas (AA) en un examen de orina (proteinuria/ hematuria). También la NC1q puede manifestarse por hematuria macroscópica, síndrome nefrítico, insuficiencia renal aguda, insuficiencia renal crónica, glomerulonefritis rápidamente progresiva, síndrome urémico-hemolítico, vasculitis o hipertensión arterial ${ }^{2-4,6-8,11,19}$.

En una de las series publicadas con 16.860 pacientes con biopsia de riñones nativos, en 61 $(0,4 \%)$ se diagnosticó una NC1q. En 36 la forma de presentación fue AA del examen de orina y en 25 un SN. La edad varió entre 1 y 67 años (media: $19,6 \pm 15,4)$. El patrón histológico a la microscopía de luz en los que se presentaron clínicamente con un SN fue: NCM en 92\% y GSFS en 8\%. En los que se presentaron con AA del examen de orina el patrón histológico fue: NCM en 64\%, GN proliferativa mesangial (GNPM) en 19\% y GSFS en $17 \%$. La fibrosis intersticial fue más frecuente en GNPM. En la microscopía de inmunofluorescencia, en ambos tipos de presentaciones clínicas, se encontraron depósitos de C1q solos o en co-dominancia con IgG, IgM o C3, en combinaciones variables. En la microscopía electrónica lo más frecuente en ambos tipos de presentaciones fue la presencia de depósitos densos mesangiales solos y ocasionalmente se observaron asociados a depósitos subendoteliales y/o subepiteliales escasos ${ }^{11}$.

En las otras 4 series publicadas que incluyeron a niños y adultos (161 en total), la proporción de los diferentes patrones histológicos varió entre $73 \%$ y $33 \%$ para NCM, $37 \%$ y $15 \%$ para GSFS y $46 \%$ y $9 \%$ para GNPM $^{3,16,17,18}$. Las edades, razas e indicaciones de $\mathrm{BpR}$ fueron muy diferentes entre las series.

Los pacientes que se presentan con un $\mathrm{SN}$ y cuyo patrón histológico es una NCM o GSFS son más jóvenes que los que se presentan con $\mathrm{AA}$ del examen de orina y cuyo patrón es una $\mathrm{GNPM}^{3}$. Estos últimos suelen tener un clearance de creatinina más bajo y más frecuentemente hematuria que los pacientes con $\mathrm{SN}$ al momento de la $\mathrm{BpR}$. La presión arterial, en cambio, no fue diferente entre los 2 grupos ${ }^{11}$.

En la inmunofluorescencia, los depósitos mesangiales de C1q en la mayoría son globales. Ocasionalmente pueden observarse en paredes capilares libres. El depósito de $\mathrm{Clq}$ asociado a IgG, IgM, IgA y C3 (“full house” en la terminología en inglés) se ha observado predominantemente en los pacientes con $\mathrm{GNPM}^{3}$. En la microscopía electrónica, la fusión pedicelar y la condensación del citoesqueleto podocitario se observa más frecuentemente en los pacientes con SN, como en este paciente. La presencia de inclusiones túbulo-reticulares en las células endoteliales debe hacer sospechar un LES, las que no existían en este paciente ${ }^{1,3}$.

La positividad de C1q en la inmunofluorescencia puede ocurrir por su unión a la porción Fc de inmunoglobulinas unidas a la superficie celular, a la laminina (proteína de la membrana basal), a complejos inmunes atrapados inespecíficamente en el mesangio como resultado del aumento en el tráfico de proteínas, a receptores presentes en la superficie celular o por unión a células apoptóticas o epítopes atrapados en el glomérulo ${ }^{12}$. Otros los han relacionado con infecciones por los virus de Epstein Barr o BK (19). La patogenia de la podocitopatía en la NClq es más incierta ${ }^{3,9}$.

Los pacientes nefróticos con NCM y depósitos de $\mathrm{Clq}$, al compararlos con enfermos con $\mathrm{SN}$ y NCM sin estos depósitos, tienen en su presentación albuminemias más bajas y mayor prevalencia de hematuria microscópica².

Los pacientes nefróticos con NClq, si bien logran obtener la remisión con corticoides, tienen recaídas frecuentes, se hacen esteroides dependientes o resistentes, debiendo utilizar inmunosupresores como ciclofosfamida, ciclosporina, tacrolimus, micofenolato o rituximab, para mantener la remisión ${ }^{2,9,12}$. Este paciente tuvo este comportamiento y debió usar varias de estas drogas para reducir las dosis de esteroides.

Los pacientes con GNPM sin SN habitualmente tienen una evolución benigna, los con GSFS tienen un curso arrastrado, pobre respuesta a esteroides y pueden desarrollar una falla renal terminal, aunque hay casos con remisión espontánea ${ }^{2,3,10}$. Algunos pacientes con $\mathrm{SN}$ y un patrón de NCM en la BpR inicial pueden desarrollar una GSFS durante su evolución ${ }^{11}$. Este paciente ha tenido una evolución con recaídas pero que no ha deteriorado su función renal en los 12 años de iniciado su tratamiento posterior al primer episodio de SN.

En pacientes sometidos a una nueva biopsia durante su evolución, se han observado tanto aumento de los depósitos de C1q a pesar de la terapia inmunosupresora, como desaparición de los depósitos con persistencia de las alteraciones podocitarias y del $\mathrm{SN}^{5,9,11}$. Este paciente tiene 
depósitos de C1q mesangiales a pesar de utilizar un esquema inmunosupresor potente por aproximadamente 5 años.

Comunicamos este caso de $\mathrm{SN}$ con las características de NC1q y su evolución durante 12 años, como un diagnóstico diferencial importante a considerar al enfrentar un SN de evolución tórpida.

\section{Referencias}

1. Jennette JC, Hipp CG. C1q nephropathy: a distinct pathologic entity usually causing nephrotic syndrome. Am J Kidney Dis 1985; 6: 103-10.

2. Wong CS, Fink CA, Baechle J, Harris AA, Staples AO, Brandt JR. C1q nephropathy and minimal change nephrotic syndrome. Pediatr Nephrol 2009; 24: 761-767.

3. Vizjak A, Ferluga D, Rozic M, Hvala A, Lindic J, Levart TK, et al. Pathology, clinical presentations, and outcomes of C1q nephropathy. J Am Soc Nephrol 2008; 19: 2237-44.

4. Malleshappa P, Ranganath P, Chaudhari AP, Ayiangar A, Lohitaksha S. C1q nephropathy presenting as acute renal failure. Saudi J Kidney Dis Transpl 2011; 22: 324-6.

5. Fülöp T, Csongrádi E, Lerant AA, Lewin M, Lewin JR. Resolution of $\mathrm{C} 1 \mathrm{q}$ deposition but noto $\mathrm{f}$ the clinical nephrotic syndrome after immunomodulating therapy in focal sclerosis. J Nephropathol 2015; 4: 54-8.

6. Maliakkal JG, Liapis H, White AJ, Ahn S-Y. Clq nephropathy in the setting of granulomatosis with polyangiitis treated with tacrolimus. Clin Kidney J 2014; 7: 499-500.

7. Kanodia KV, Vanikar AV, Shah PR, Kute VB, Feroz A, Suthar K, et al. C1q nephropathy with hemolytic uremic syndrome. Saudi J Kidney Dis Transpl 2012; 23: 556-8.

8. Bhowmik DM, Jain S, Dinda AK, Sharma A, Mahajan S, Agarwal SK. C1q nephropathy presenting as nephritic-nephrotic syndrome. Saudi J Kidney Dis Transpl
2011; 22: 561-3.

9. Muorah M, Sinha MD, Horsfield C, O’Donnell PJ. C1q nephropathy: a true immune complex disease or a immunologic epiphenomenon? NDT Plus 2009; 2: 285-91.

10. Panuncio A, Alba A, Bacigalupe C, Caorsi H, Schwedt E, Otatti G, et al. Nefropatía C1q en un paciente de 17 años. Caso clínico. Rev Med Chile 2008; 136: 1307-10.

11. Hisano S, Fukuma Y, Segawa Y, Niimi K, Kaku Y, Hatae $\mathrm{K}$, et al. Clinicopathologic correlation and outcome of Clq nephropathy. Clin J Am Soc Nephrol 2008; 3: 1637 43.

12. Wenderfer SE, Swinford RD, Braun MC. C1q nephropathy in the pediatric population: pathology and pathogenesis. Pediatr Nephrol 2010; 25: 1385-96.

13. Malleshappa P. C1q nephropathy-unity in diversity. J Renal Inj Prev 2013; 2: 117-8.

14. Devasahayam J, Erode-Singaravelu G, Bhat Z, Oliver T, Chandran A, Zeng X, et al. C1q nephropathy: the unique underrecognized pathological entity. Anal Cell Pathol (Amst) 2015; 4090413. doi: 10.1155/2015/490413. Epub 2015 Nov 10.

15. Malleshappa P, Vankalakunti M. Diverse clinical and histology presentation in C1q nephropathy. Nephro Urol Mon 2013; 5: 787-91.

16. Gunasekara VN, Sebire NJ, Tullus K. Clq nephropathy in children: clinical characteristics and outcome. Pediatr Nephrol 2014; 29: 407-13.

17. Fukuma Y, Hisano S, Segawa Y, Niimi K, Tsuru N, Kaku $\mathrm{Y}$, et al. Clinicopathologic correlation of C1q nephropathy in children. Am J Kidney Dis 2006; 47: 412-8.

18. Said SM, Cornell LD, Valeri AM, Sethi S, Fidler ME, Cosio FG, et al. C1q deposition in the renal allograft: a report of 24 cases. Mod Pathol 2010; 23: 1080-8.

19. Markowitz GS, Schwimmer JA, Stokes MB, Nasr S, Seigle RL, Valeri AM, et al. C1q nephropathy: A variant of focal segmental glomerulosclerosis. Kidney Int 2003; 64: 1232-40. 